\title{
PSORÍASE E SEUS TRATAMENTOS: UMA REVISÃO DA LITERATURA
}

\author{
Letícia Borin Silva'; Camila Franco² \\ 1 Graduada do Curso de Farmácia da Universidade Franciscana-UFN, Rua dos \\ Andradas, 1614. CEP: 97010-032. Santa Maria, RS. leticia booriin@hotmail.com. \\ 2 Professora Doutora do Curso de Farmácia da Universidade Franciscana-UFN, Rua \\ dos Andradas, 1614. CEP: 97010-032. Santa Maria, RS. cf@ufn.edu.br.
}

\section{RESUMO}

A psoríase é uma patologia dermatológica, inflamatória, crônica, autoimune e de manifestação multifatorial, entretanto, não é contagiosa. Objetivou-se realizar uma revisão sistemática sobre a psoríase e seus tratamentos por meio de uma revisão de artigos publicados nos últimos 5 anos em bancos eletrônicos utilizando DeCS. São vários os protocolos terapêuticos para psoríase, sendo escolhidos conforme a gravidade do caso clínico. As terapêuticas tópicas com corticoides são empregadas em casos iniciais enquanto terapias sistêmicas são utilizadas em casos graves. A fototerapia reduz tempo de tratamento e os efeitos adversos das terapêuticas isoladas, sendo que a associação apresenta bons resultados. Dentre os medicamentos inovadores destacam-se os imunobiológicos como adalimumabe e secuquinumabe que estão sendo utilizados inclusive na prescrição do sistema único de saúde e, que demonstram os mais rápidos e melhores resultados no controle da patologia. Conclui-se que o adalimumabe ofereceu menor custo-resposta e o secuquinumabe apresentou melhor resposta clínica.

Palavras-chave: Eficácia, Segurança, Terapêutica.

Eixo Temático: Atenção Integral e Promoção à Saúde (AIPS).

\section{INTRODUÇÃO}

A psoríase é uma patologia dermatológica inflamatória crônica, autoimune e de manifestação multifatorial, embora não contagiosa. Caracterizada pela ativação imune inapropriada mediada por linfócitos $\mathrm{T}$, que aumentam a liberação de citocinas pró-inflamatórias, ocorre hiperproliferação de queratinócitos na epiderme e espessamento da camada córnea (hiperqueratose) (BAPTISTA, 2014; CONITEC, 2019a). 
EDUCAÇÃO, SAÚDE

ETECNOLOGIA

26 A 28 DE OUTUBRO DE 2021

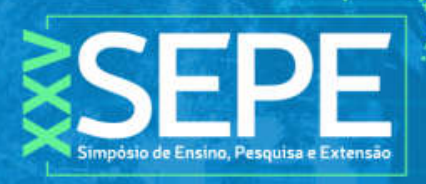

QUFN

A manifestação mais comum (80\%) é a psoríase vulgar ou em placas que manifesta-se por placas secas eritemo-escamosas, avermelhadas com escamas prateadas ou esbranquiçadas, em áreas de traumas, podendo acometer todo o corpo. Outros tipos de psoríase encontram-se na Figura 1.

Figura 1: Tipos de psoríase e sua média de incidência.

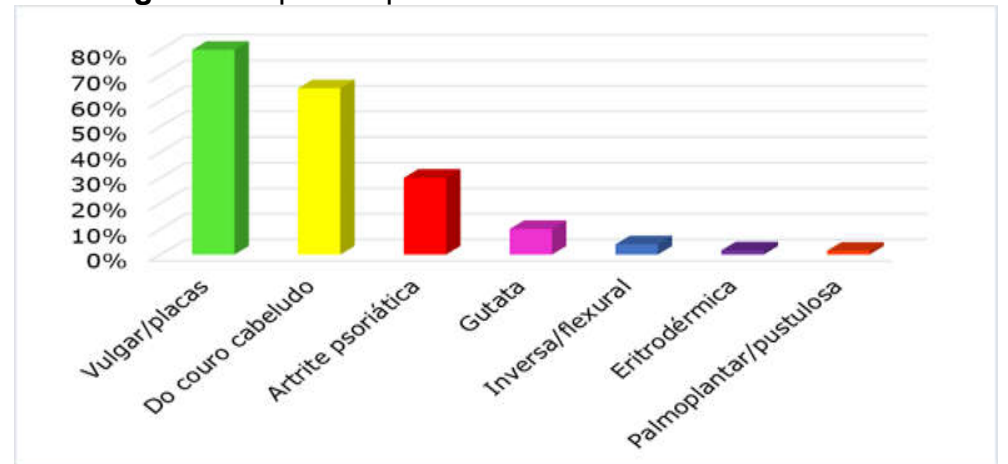

Fonte: Elaborada pelo autor, com base nas referências (CRUZ, 2011; ROMITI, 2009; SILVA, 2016; SOARES, 2013; SOUSA, 2018).

O tratamento visa obter períodos prolongados de alívio dos sintomas por meio do emprego de medicamentos de aplicação tópica, os quais afetam a proliferação e produção de mediadores inflamatórios envolvidos na patogênese; ou fototerapia que emprega a radiação ultravioleta (UV), que pode ser associada ao psoraleno (ex. PUVA) e, ocasiona uma imunossupressão local, a redução da hiperproliferação epidérmica e a apoptose de linfócitos T (DUARTE, 2009; MARTINS, 2009). É possível ainda associar ambas ou não e, ainda, utilizar medicamentos sistêmicos orais ou injetáveis (ROMITI, 2009). O tratamento é iniciado por medicamentos tópicos e fototerapia, mas quando os pacientes não respondem ou são intolerantes, faz-se uso dos sistêmicos.

Novos tratamentos vêm surgindo, como agentes biológicos (sistêmicos), que envolvem um grupo de fármacos que integram o anti-fator de necrose tumoral alfa (Anti-TNFa) e, incluem: anticorpos monoclonais, proteínas de fusão ou antiinterleucinas 12 e 23 (CONITEC, 2019a,b). Dessa forma, o objetivo desta pesquisa foi a realização de um levantamento bibliográfico das publicações cientificas existentes e atualizadas sobre a psoríase, tratamentos e inovações terapêuticas considerando eficácia e segurança. 


\section{METODOLOGIA}

Este estudo aborda uma revisão sistemática qualitativa descritiva sobre a psoríase utilizando descritores em Ciências da Saúde (DeCS): "psoríase, terapêutica, eficácia e segurança" consultados artigos disponíveis em bancos de dados eletrônicos como Google acadêmico, Pubmed e Science Direct e, publicados nos últimos 5 anos, sendo excluidos trabalhos que não contivessem metodologia clara ou não trouxessem informações sobre eficácia e/ou segurança dos tratamentos. Após a coleta e avaliação das informações, os dados foram organizados sistematicamente, promovendo discussões sobre o tema.

\section{RESULTADOS E DISCUSSÕES}

A escolha do tipo de tratamento depende de cada caso, conforme a dimensão e o grau de severidade (ROMITI, 2009). A primeira escolha é o tratamento tópico, apesar da adesão ser baixa devido a aplicação desagradável (CRUZ, 2011; SILVA, 2016). Na Tabela 1 são apresentados os tratamentos tópicos.

Tabela 1-Medicações tópicas para psoríase.

\begin{tabular}{l|lll}
\hline Tipo & Indicação & Terapêutica & Referências \\
\hline $\begin{array}{l}\text { Queratolíticos } \\
\text { (ácido salicílico e }\end{array}$ & $\begin{array}{l}\text { Psoríase em } \\
\text { placas e do couro } \\
\text { uréia) }\end{array}$ & $\begin{array}{l}\text { Concentração de 3\% a } \\
\text { cabeludo apticado a noite e }\end{array}$ & $\begin{array}{l}\text { MART, 2009; } \\
\text { removido no banho no dia } \\
\text { seguinte }\end{array}$ \\
\hline
\end{tabular}




\begin{tabular}{|c|c|c|c|}
\hline \multicolumn{2}{|c|}{$\begin{array}{l}\text { EDUCAÇÃO, SAÚDE } \\
\text { ETECNOLOGIA } \\
26 \text { A } 28 \text { DE OUTUBRO DE } 2021\end{array}$} & & $\begin{array}{r}\text { ISSN: } 2316-9745 \\
\text { TRABALHO COMPLETO }\end{array}$ \\
\hline $\begin{array}{l}\text { Alcatrão de } \\
\text { carvão } \\
\text { (coaltar) }\end{array}$ & $\begin{array}{l}\text { Psoríase em } \\
\text { placas }\end{array}$ & $\begin{array}{l}\text { Concentração de } 1 \text { a 5\%, } \\
\text { aplicado } 1 x \text { ao dia }\end{array}$ & $\begin{array}{lr}\text { CRUZ, 2011; } & \text { SOCIEDADE } \\
\text { BRASILEIRA } & \\
\text { DERMATOLOGIA, } 2012 . & \text { DE }\end{array}$ \\
\hline Antralina & $\begin{array}{l}\text { Psoríase vulgar, } \\
\text { ungueal e do } \\
\text { couro cabeludo }\end{array}$ & $\begin{array}{l}\text { Concentração de } 0,05 \text { a } \\
3 \% \text { mantendo produto } \\
\text { até } 12 \mathrm{~h} \text { de contato e de } \\
10 \text { minutos a } 1 \mathrm{~h} \text { de } \\
\text { contato nas maiores } \\
\text { concentrações }\end{array}$ & SOUSA, 2018. \\
\hline $\begin{array}{l}\text { Corticosteróides } \\
\text { (propionato de } \\
\text { clobetasol) }\end{array}$ & $\begin{array}{l}\text { Psoriase em } \\
\text { placas moderada } \\
\text { a grave }\end{array}$ & $\begin{array}{l}\text { Concentração de } 0,05 \% \\
\text { aplicado } 1 \times \text { ao dia }\end{array}$ & $\begin{array}{l}\text { SOCIEDADE BRASILEIRA DE } \\
\text { DERMATOLOGIA, } \\
\text { SUKAROVSKA, } 2006\end{array}$ \\
\hline $\begin{array}{l}\text { Análogos de } \\
\text { vitamina } D_{3} \\
\text { (calcipotriol) }\end{array}$ & $\begin{array}{lr}\text { Psoríase do couro } \\
\text { cabeludo r e } \\
\text { psoríase das } \\
\text { unhas }\end{array}$ & 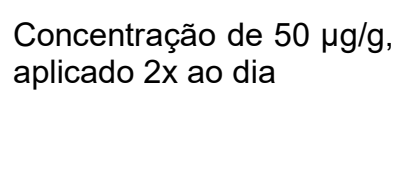 & $\begin{array}{l}\text { SOCIEDADE BRASILEIRA DE } \\
\text { DERMATOLOGIA, } 2012\end{array}$ \\
\hline $\begin{array}{l}\text { Retinóides } \\
\text { (tazaroteno) }\end{array}$ & $\begin{array}{l}\text { Psoríase crônica } \\
\text { e em placas }\end{array}$ & $\begin{array}{l}\text { Concentração de } 0,05 \% \text { e } \\
\text { a } 0,1 \% \text {, aplicado até } 2 x \\
\text { ao dia }\end{array}$ & SILVA, 2014 \\
\hline Tacrolimus & $\begin{array}{l}\text { Psoríase em } \\
\text { placas leve a } \\
\text { moderada }\end{array}$ & $\begin{array}{l}\text { Concentração de } 0,03 \text { e } \\
0,1 \% \text {, aplicado até } 2 x \text { ao } \\
\text { dia }\end{array}$ & MALECIC, 2016 \\
\hline
\end{tabular}

Fonte: Elaborada pelo autor.

Os queratolíticos agem eliminando as escamas, mas deve-se evitar uso contínuo de ácido salicílico devido ao potencial nefrotóxico e neurotóxico (SOUSA, 2018). O alcatrão de carvão (coaltar) possui eficácia moderada aumentada quando associado à radiação UVB, mas desaconselha-se uso na psoríase pustular e eritrodermica e, em zonas sensíveis devido a potencial de foliculite, eczema e fototoxicidade (CRUZ, 2011). A antralina é anti-inflamatória dose-dependente, mas pode gerar eritema e sensação de queimadura (CRUZ, 2011; SOUSA, 2018). Os corticosteróides possuem eficácia moderada a alta, mas efeitos colaterais com o uso prolongado, como: atrofia e branqueamento cutâneo, eritema, dermatite, erupções acneiformes, infecções, catarata e glaucoma (CONITEC, 2019b; SUKAROVSKA, 2006). Os análogos de vitamina $D_{3}$ são uma alternativa para psoríase tópica, mas geram irritação local, podendo apresentar prurido, eritema, ardor, foliculite e alterações da pigmentação (CRUZ, 2011; SOCIEDADE BRASILEIRA DE DERMATOLOGIA, 2012). Os retinoides possuem eficácia observada em duas semanas, mas são contraindicados em grávidas devido a irritação local. E, o 
tacrolimus é eficaz para psoríase inversa, mas pode causar prurido leve, sensação de calor no local de aplicação, o qual diminui gradativamente (MALECIC, 2016).

Alguns produtos naturais podem ser utilizados como: camomila-romana (Chamaemelum nobile) com ação imunoestimulante, calmante e analgésica; a babosa (Aloe vera L.) com ação analgésica, antipruriginosa, antialérgica, cicatrizante e antiinflamatória; a curcumina (Curcuma Longa) com ação antiinflamatória, antiproliferativa e pró-apoptótica sobre queratinócitos e; amêndoas doces (Prunus amygdalis var. Dulcis) a qual é antiinflamatória e antipruriginosa (SILVA et al., 2011). Esses protudos apresentam melhora de sintomas (camomila e óleo de amêndoas) até a resolução das lesões (com babosa e curcumina) (SILVA, 2011). Apenas para a babosa são relatados efeitos adversos: vermelhidão, ardor e dermatite generalizada.

A fototerapia é indicada quando os tratamentos tópicos são insuficientes no controle da psoríase ou para lesões demasiadamente grandes e dispersas, usada para todos os tipos de psoríase por possuir ação anti-inflamatória, imunossupressora e antiproliferativa, causando efeito sistêmico mínimo ou inexistente (DUARTE, 2006; SOCIEDADE BRASILEIRA DE DERMATOLOGIA, 2012). A aplicação pode ser realizada com irradiação UVA $(400-320 \mathrm{~nm})$ capaz de atingir a derme profunda ou UVB $(320$ - $290 \mathrm{~nm}$ ) que atinge somente a epiderme e, pode ser associada com medicamentos como retinóides, metotrexato, ciclosporina, antralina e corticosteroides, os quais levam a um rápido controle das dermatoses com doses menores dos medicamentos. Na Tabela 2 estão listadas as modalidades de fototerapia, sua indicação e terapêutica disponível (DUARTE, 2006).

Tabela 2- Modalidades de fototerapia sua indicação e terapêutica disponível.

\begin{tabular}{|c|c|c|c|}
\hline Tipo & Indicação & Terapêutica & Referências \\
\hline $\begin{array}{l}\text { UVB de banda } \\
\text { estreita (NB) }\end{array}$ & $\begin{array}{l}\text { Psoríase gutata e } \\
\text { de placas finas }\end{array}$ & $\begin{array}{l}\text { Dose inicial: } 100 \text { mJ/cm }{ }^{2} \text {, sendo } \\
\text { aumentada gradativamente para mais } \\
40 \mathrm{~mJ} / \mathrm{cm}^{2} \text {, de } 2 \text { a } 3 x \text { por semana } \\
\text { durante } 3 \text { a } 6 \text { meses }\end{array}$ & $\begin{array}{l}\text { SOCIEDADE } \\
\text { BRASILEIRA DE } \\
\text { DERMATOLOGIA, } \\
2012\end{array}$ \\
\hline PUVA tópico & $\begin{array}{l}\text { Psoríase palmo- } \\
\text { plantar ou couro } \\
\text { cabeludo }\end{array}$ & $\begin{array}{l}\text { Dose inicial UVA: } 0,12 \text { a } 0,5 \mathrm{~J} / \mathrm{cm}^{2} \text {, } \\
\text { aumentando a } 0,12-0,25 \mathrm{~J} / \mathrm{cm}^{2} \text { a } \\
\text { cada sessão, realizadas } 2 \text { a } 3 x \text { por } \\
\text { semana. Pode levar até } 30 \text { sessões }\end{array}$ & $\begin{array}{l}\text { DUARTE, } 2006 ; \\
\text { SOUSA, 2018. }\end{array}$ \\
\hline
\end{tabular}




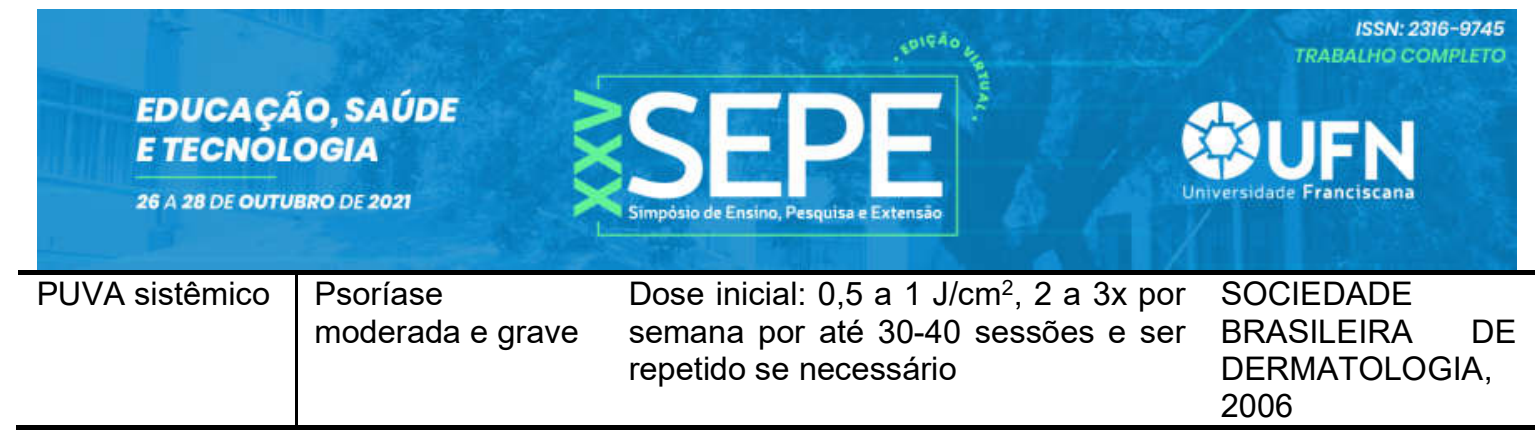

Fonte: Elaborada pelo autor.

A radiação UVB NB apresenta eficácia a partir da quinta sessão, sendo seguro, podendo ser indicada na gravidez e uso em crianças (DUARTE, 2009; SOCIEDADE BRASILEIRA DE DERMATOLOGIA, 2012). Somente é contraindicada à pacientes com fotossensibilidade e antecedentes de melanoma ou cancro da pele (SOUSA, 2018).

Entretanto, quando terapias tópicas e fototerapia não são suficientemente eficazes, faz-se o emprego de terapia sistêmica recomendada em casos moderados a graves (20\% a 30\%) (CONITEC, 2019a,b; TEIXEIRA, 2018). A Tabela 3, apresenta os três tratamentos sistêmicos aprovados pela Sociedade Brasileira de Dermatologia.

Tabela 3- Tratamentos sistêmicos aprovados para a psoríase: metotrexato, acitretina e ciclosporina.

\begin{tabular}{|c|c|c|c|}
\hline $\begin{array}{l}\text { Agente } \\
\text { sistêmico }\end{array}$ & Tipo de psoriase & Terapêutica & Referências \\
\hline Metotrexato & $\begin{array}{l}\text { Artrite psoriática e } \\
\text { psoríase } \\
\text { eritrodérmica ou } \\
\text { pustular }\end{array}$ & $\begin{array}{l}\text { Comprimidos de } 2,5 \mathrm{mg} \text { ou solução } \\
\text { injetável de } 2 \mathrm{~mL} \text { contendo } 50 \mathrm{mg} \\
\text { da droga ( } 25 \mathrm{mg} / \mathrm{mL}) \text {. Alcançadas } \\
\text { as respostas clínicas reduz-se as } \\
\text { doses. Duração média do } \\
\text { tratamento: } 2 \text { a } 4 \text { meses }\end{array}$ & $\begin{array}{l}\text { ARRUDA, } \\
\text { BAPTISTA, } 2014\end{array}$ \\
\hline Ciclosporina & $\begin{array}{l}\text { Psoríase vulgar } \\
\text { moderada a grave, } \\
\text { pustulosa e na } \\
\text { artrite psoriática }\end{array}$ & $\begin{array}{l}\text { Dose inicial: cápsula de } 2,5 \\
\text { mg/kg/dia, dividida em duas } \\
\text { administrações. Pode aumentar } \\
\text { gradualmente, a cada } 2 \text { a } 4 \\
\text { semanas, em } 0,5 \text { a } 1 \mathrm{mg} / \mathrm{Kg} / \mathrm{dia} \text {, } \\
\text { até a dose máxima de } 5 \mathrm{mg} / \mathrm{Kg} / \mathrm{dia} \\
\text { Duração média do tratamento: } 12 \\
\text { semanas }\end{array}$ & $\begin{array}{l}\text { SOCIEDADE } \\
\text { BRASILEIRA } \\
\text { DERMATOLOGIA, } 2012\end{array}$ \\
\hline Acitretina & $\begin{array}{l}\text { Formas graves de } \\
\text { psoríase } \\
\text { eritrodérmica e } \\
\text { pustular, localizada } \\
\text { ou generalizada }\end{array}$ & $\begin{array}{l}\text { Cápsulas de } 10 \text { e } 25 \mathrm{mg} \\
\text { Duração do tratamento: } 3 \text { a } 6 \\
\text { meses }\end{array}$ & $\begin{array}{l}\text { ARRUDA, } \\
\text { CONITEC, 2019b }\end{array}$ \\
\hline
\end{tabular}

Fonte: Elaborada pelo autor. 


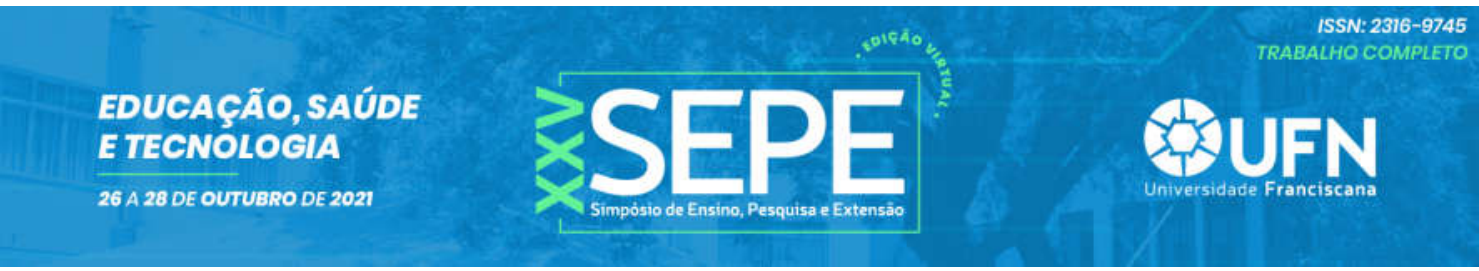

O metotrexato tem excelente eficácia, mas sua toxicidade hepática cumulativa impede o uso prolongado (ARRUDA, 2004a; BAPTISTA, 2014). A ciclosporina exibe melhoras rápidas em 80 a 90\% dos pacientes, sendo que, 50 a 70\% obtiveram remissão parcial (índice de extensão e gravidade da psoríase - PASI 75) e 30 a 50\% remissão completa (PASI 90), após 12 semanas. Seus efeitos adversos são: nefrotoxicidade, hipertensão arterial sistêmica e risco de malignidade tempo e dosedepentendes (SOCIEDADE BRASILEIRA DE DERMATOLOGIA, 2012). Já a acitretina expressa a remissão total ou uma melhora em $80 \%$ do quadro clínico em $100 \%$ nas formas pustulosas, $83 \%$ nas eritrodérmicas e $76,5 \%$ em placa. Os efeitos adversos compreendem secura da boca, olhos e nariz, fraqueza capilar, hipercolesterolemia, sendo reversíveis quando é descontinuada (ARRUDA, 2004a;CONITEC, 2019b).

Além dos tratamentos acima, existem medicamentos inovadores, porém de custo elevado, o que torna o uso restrito e, são apresentados na Tabela 4.

Tabela 4- Medicamentos inovadores usados na terapia biológica.

\begin{tabular}{|c|c|c|}
\hline Princípio ativo & Terapêutica & Referências \\
\hline Infliximabe & $\begin{array}{l}\text { Administração IV - Dose: } 5 \text { mg/kg. Repete-se } \\
\text { administração de } 2 \text { e } 6 \text { semanas após a primeira } \\
\text { dose }\end{array}$ & $\begin{array}{l}\text { SOCIEDADE BRASILEIRA } \\
\text { DE } \quad \text { DERMATOLOGIA, } \\
2012 .\end{array}$ \\
\hline Adalimumabe & $\begin{array}{l}\text { Administração SC - Dose inicia: } 80 \text { mg, seguida de } \\
\text { uma dose de } 40 \mathrm{mg} \text {, uma semana após. A cada } 14 \\
\text { dias, administrar } 40 \mathrm{mg} \text { por até } 16 \text { semanas }\end{array}$ & CONITEC, $2018 a$ \\
\hline Ustequinumabe & $\begin{array}{l}\text { Administração SC - Dose: } 45 \mathrm{mg} \text { nas semanas } 0 \text { e } 4 \\
\text { e repetir a cada } 12 \text { semanas. Interromper tratamento } \\
\text { se não houver resposta em até } 28 \text { semanas. } \\
\text { Pacientes com peso corpóreo maior que } 100 \mathrm{Kg} \\
\text { pode ser utilizada a dose de } 90 \mathrm{mg} \text {. }\end{array}$ & CONITEC, 2018a,b \\
\hline Etanercepte & $\begin{array}{l}\text { Administração SC - Dose: } 25 \mathrm{mg}, 2 x \text { por semana ou } \\
50 \mathrm{mg}, 1 \mathrm{x} \text { por semana ou } 50 \mathrm{mg}, 2 \mathrm{x} \text { por semana até } \\
12 \text { semanas, seguida de uma dose de } 25 \mathrm{mg}, 2 \mathrm{x} \text { por } \\
\text { semana ou } 50 \mathrm{mg} \text {, 1x por semana. Tratamento } \\
\text { máximo até } 24 \text { semanas. }\end{array}$ & CONITEC, 2018a \\
\hline Ixequizumabe & $\begin{array}{l}\text { Administração SC - Dose: } 160 \text { mg (duas injeções de } \\
80 \mathrm{mg} \text { ) na } 1^{\text {a }} \text { semana, seguida por uma injeção de } \\
80 \mathrm{mg} \text { nas semanas } 2,4,6,8,10 \text { e } 12 \text { e então, } 80 \\
\text { mg a cada } 4 \text { semanas. Tratamento por } 16 \text { a } 20 \\
\text { semanas. }\end{array}$ & CONITEC, 2020a \\
\hline
\end{tabular}




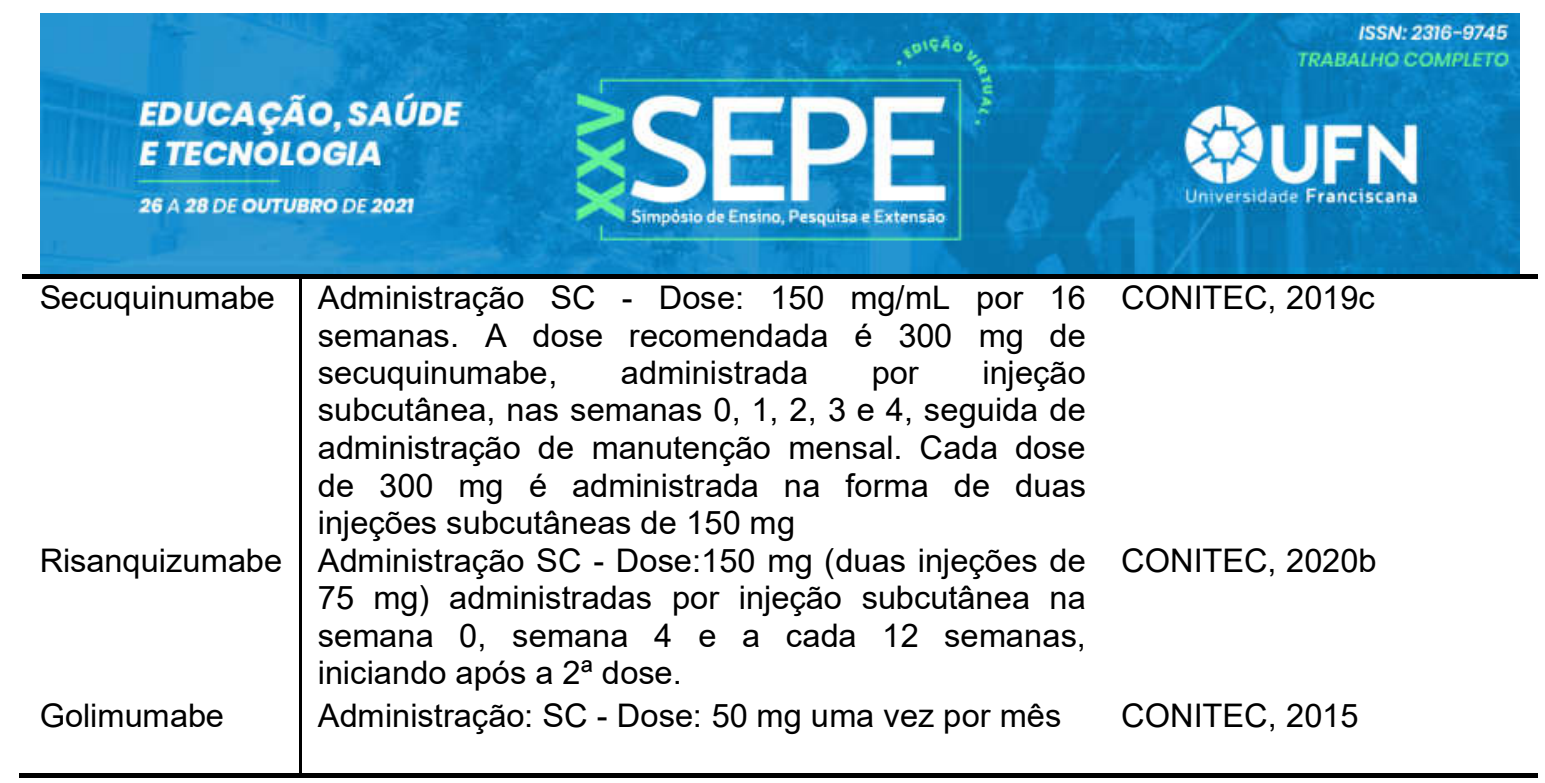

Fonte: Elaborada pelo autor.

O infliximabe melhora os sintomas e é bem tolerado porém, efeitos adversos (dispinéia e hipotensão) podem perdurar até duas horas após a infusão, devendo ser controlados em ambiente hospitalar (ARRUDA, 2004b). Adalimumabe em ensaio com 1212 pacientes demonstrou que 71\% atingiram o PASI 75 (CONITEC, 2012), assim como o etanercepte que resultou em PASI 75 para $49 \%$ dos pacientes (ARRUDA, 2004b; TEIXEIRA, 2018;). O ustequinumabe mostrou ser eficaz no tratamento de psoríase moderada a grave (CONITEC, 2018b). E o ixequizumabe foi mais eficaz com o uso a cada duas semanas de forma superior ao adalimumabe e ustequinumabe (CONITEC, 2020a). O secuquinumabe demonstrou ter eficácia alta por apresentar pele sem lesões ou quase sem lesões (PASI $\geq 90)$ (CONITEC, 2019c). O risanquizumabe foi seguro e eficaz em psoríase de placa crônica moderada a grave, artrite psoriásica e doença de Crohn (CONITEC, 2020b; WITJES, 2020). Já o golimumabe foi eficaz para psoríase em placas e artrite psoríasica, da mesma forma que adalimumabe, infliximabe e etanercepte, tendo como vantagem a aplicação apenas uma vez ao mês (CONITEC, 2015). Esses imunobiológicos apresentam, de forma geral, reações adversas no local da injeção e infecções leves em vias aéreas e no trato genito-urinário, podendo haver micoses superficiais, cefaléia, náusea, vômito, diarréia, mialgia e artralgia.

Como levantamento desse estudo foram considerados 50 artigos, que abordavam os tratamentos para psoríase, sendo 10\% empregados os de uso tópico e $10 \%$ produtos naturais, $6 \%$ fototerapia, $4 \%$ os de uso sistêmico, $27 \%$ emprego dos 
EDUCAÇÃO, SAÚDE

ETECNOLOGIA

26 A 28 DE OUTUBRO DE 2021

biológicos e $37 \%$ medicamentos de uso geral, ou seja, que abordam diferentes tipos de tratamento.

Embora sem cura, os tratamentos aliviam os sintomas e melhoram a qualidade de vida do paciente. São de primeira escolha os tópicos, não havendo um medicamento preferencial. Constata-se que corticosteroides tópicos são bem tolerados e eficazes e, muitas vezes são substituídos por análogos de vitamina $D_{3}$ ou retinóides. Dentre os produtos naturais destaca-se o Aloe vera, por apresentar excelente melhora ou completa resolução das lesões (CRUZ, 2011). A fototerapia é benéfica, embora ainda deva ser avaliada em termos de magnitude, duração e segurança a longo prazo. Associada a medicamentos, diminui o tempo de tratamento e efeitos colaterais, causando o alivio de sintomas por período prolongado. O metotrexato, acitretina e ciclosporina demonstraram eficácia a longo prazo, entretanto, apresentaram risco de toxicidade hepática cumulativa, o que impede o uso continuado (ARRUDA, 2004b; SOCIEDADE BRASILEIRA DE DERMATOLOGIA, 2006). Imunobiológicos inovadores revolucionaram e aumentaram o leque terapêutico em casos de psoríase grave, obtendo eficácia e segurança, com perfil remissivo superior aos demais tratamentos.

\section{CONCLUSÃO}

Conclui-se que medicamentos tópicos são a primeira escolha para tratamento da psoríase, seguido pelos sistêmicos, de acordo com a gravidade do caso. Há a necessidade de pesquisas de novos fármacos com mecanismos de ação capazes de modular o sistema imunológico e controlar a progressão ou promover a remissão da doença. Mais próximo dessa realidade temos os imunobiológicos, inibidores de fator de necrose turmoais (TNFa, TNFß) e de interleucinas (IL-17A e IL- 12/23). Destes, o Adalimumabe destaca-se após a recomendação da CONITEC (2018a) para primeira escolha após a falha da terapia padrão, seguido por secuquinumabe, inclusive, estes medicamentos estão sendo incorporados no âmbito do SUS. Considerou-se que o adalimumabe ofereceu menor custo-resposta e o secuquinumabe apresentou melhor resposta clínica (CONITEC, 2018a). 


\section{AGRADECIMENTOS}

Os autores agradecem a Universidade Franciscana pela infraestrutura e e apoio no desenvolvimento desta pesquisa.

\section{REFERÊNCIAS}

Arruda L; Martins GA. Tratamento sistêmico da psoríase - Parte I: metotrexato e acitretina. An Bras Dermatol., v. 79, n. 4, p.393-411. 2004a.

Arruda L; Ypiranga S; Martins GA. Systemic treatment of psoriasis -Part II: Biologic immunomodulator agents. An bras Dermatol. v. 79, n. 4, p. 393-408. 2004b.

Baptista SAP; Piloto JADR. O uso do metotrexato na psoríase. Brazilian journal of surgery and clinical research-bjscr, v. 8, n. 2, p. 54-58, 2014.

Conitec., Ministério da Saúde. Protocolo Clínico e Diretrizes Terapêuticas Psoríase. 2019a.

Conitec, Ministério da Saúde. Relatório de Recomendaçao: Medicamentos Biológicos para o tratamento da Psoríase moderada a grave em adultos. 2012.

Conitec, Ministério da Saúde. Ustequinumabe para o tratamento de pacientes adultos com artrite psoriásica ativa que apresentaram resposta inadequada aos medicamentos modificadores do curso da doença. Relatório Recom $\mathrm{n}^{\circ}$ 337. 118 p. 2018 b.

Conitec, Ministério da Saúde. Relatório de recomendação: Ixequizumabe para tratamento de pacientes adultos com psoríase moderada a grave, que tenham apresentado falha terapêutica, contraindicação ou intolerância ao adalimumabe. 65p. 2020a.

Conitec, Ministério da Saúde. Psoríase moderada a grave Secuquinumabe $\left(\right.$ Cosentyx $\left.{ }^{\circledR}\right)$ para psoríase moderada a grave. 2019c.

Conitec, Ministério da Saúde. Risanquizumabe para tratamento da psoríase em placas de moderada a grave. 57p. 2020b.

Conitec, Ministério da Saúde. Relatório para a sociedade: informações sobre recomendações de incorporação de medicamentos e outras tecnologias no SUS. 15 p. 2015.

Conitec, Ministério da Saúde. Protocolo Clínico e Diretrizes Terapêuticas: Psoríase. 
Relatório de recomendação. 105 p. 2019 b.

Conitec, Ministério da Saúde. Adalimumabe, etanercepte, infliximabe, secuquinumabe e ustequinumabe para psoríase moderada a grave. 2018a.

Cruz MJ; Magina S; Baudrier T; Azevedo F. Tratamento da psoríase em idade pediátrica - parte i: tratamento tópico. Soc Port Dermatol Venereol. v. 69, n. 1, p. 25-31. 2011.

Duarte I; Bedrikow RB; Kobata C. Fototerapia na Psoríase. In: Consenso Brasileiro De Psoríase 2009. Sociedade Brasileira De Dermatologia Fotototerapia. p. 55-61. 2009.

Duarte I; Buense R; Kobata C. Fototerapia. An Bras Dermatol. v. 81, n. 1, p. 74-82. 2006.

Malecic N; Young H. Tacrolimus for the management of psoriasis: Clinical utility and place in therapy. Psoriasis Targets Ther. v. 6, p. 153-63. 2016.

Martins GA; Chaul A. Tratamento tópico da psoríase - Consenso brasileiro de psoríase e guias de tratamento. Sociedade brasileira de dermatologia. Rio de janeiro: sbd; 2009.

Romiti R; Maragno L; Arnone M; Takahashi MDF. Psoríase na infância e na adolescência. An Bras Dermatol. v. 84, n. 1, p. 09-20. 2009.

Silva RIABP da. Psoríase: Evolução farmacoterapêutica e risco acrescido de desenvolvimento de certas neoplasias. 2014. 101 p. Dissertação, Mestrado em Ciências Farmacêtuicas. Fac Ciências da Saúde. Universidade Fernando Pessoa. Porto, 2014.

Silva BMB da. Psoríase: Classificação Clinica e tratamento topico. Cent Univ Teresa D’Ávila - UNIFATEA. v. 13, n. 24, p. 51-69. 2016.

Silva BS; Amaral MO; Vargas TS; Silva AG. Substâncias bioativas de origem vegetal no tratamento da psoríase. Nat line v. 9, n. 3, p. 124-8. 2011.

Soares TP. Nanocosmética à base de produtos naturais aplicada à Psoríase. 2013. 69 p. Dissertação. Bragança: instituto politécnico de bragança e universidade de salamanca; Bragança, 2013.

Sociedade Brasileira de Dermatologia. Consenso Brasileiro de Psoríase. Guias de Avaliação e Tratamento. p. 172. 2012. 
Sociedade Brasileira de Dermatologia. Consenso Brasileiro de Psoríase - Guias de

Tratamento. Rio de Janeiro: Sociedade Brasileira de Dermatologia, 108 p. 2006.

Sousa CC. A psoríase. 2018. 62 p. Dissertação, Mestrado em Ciências Farmacêuticas. Porto: pós-graduação, Universidade Fernando Pessoa; Porto, 2018.

Sukarovska BG; Lipozenčić J. Topical management of psoriasis - Corticosteroids and sparing corticosteroid therapy. Acta Dermatovenerologica Croat. v. 14, n. 3 , p. 188-96. 2006.

Teixeira, F. J. M. Biológicos no tratamento da psoríase - presente e futuro. 2018. 71p. Dissertação. Coimbra: Faculdade de Medicina da Universidade de Coimbra; Coimbra, 2018.

Witjes H, Khatri A, Diderichsen PM, Mandema J, Othman AA. Meta-Analyses of Clinical Efficacy of Risankizumab and Adalimumab in Chronic Plaque Psoriasis: Supporting Evidence of Risankizumab Superiority. Clin Pharmacol Ther. v. 107, n. 2, p. 435-42. 2020. 